\title{
How Healthy and Unhealthy Values Predict Hedonic and Eudaimonic Well-Being: Dissecting Value-Related Beliefs and Behaviours
}

\author{
Agnieszka Bojanowska' ${ }^{10} \cdot$ Łukasz D. Kaczmarek $^{2} \mathbb{D}$
}

Accepted: 12 April 2021 / Published online: 21 April 2021

(c) The Author(s) 2021

\begin{abstract}
Despite a strong link between values and well-being, little is known about dimensions moderating this link, e.g., whether individuals who act upon their values experience greater well-being for healthy values (e.g., self-transcendence) and lower well-being for unhealthy values (e.g., self-enhancement). Moreover, research on values and value-related behavior has rarely accounted for hedonic and eudaimonic well-being at the same time. Thus, we aimed to examine how values, value-related behaviors, and their interaction relate to hedonic and eudaimonic well-being. We expected that 'healthy' values would correspond with higher well-being and unhealthy values with lower well-being. A community sample representative of young adults $(N=1161)$ reported their values, value-related behavior, and well-being. We found that for most values, behavior was an additional independent predictor of well-being related either to even more (e.g., self-transcendence) or less (e.g., selfenhancement) positive outcomes. For some values, behavior moderated the link between beliefs and well-being by boosting (self-transcendence) or suppressing it (conservation). We also found different links between hedonic versus eudaimonic well-being, e.g., selfenhancement and openness to change. This study presents the importance of asking about value-related beliefs, behaviors, their congruence, and specific facets of well-being when analyzing the value and well-being link. We conclude that some values are best for wellbeing when they remain passive, with little accompanying behaviors (self-enhancement or conservation), whereas others provide more benefits when individuals act upon them (self-transcendence).
\end{abstract}

Keywords Schwartz's values · Eudaimonic well-being $\cdot$ Hedonic well-being $\cdot$ Valueexpressive behaviors

Agnieszka Bojanowska

abojanowska@swps.edu.pl

1 Department of Psychology, SWPS University of Social Sciences and Humanities, Warsaw, Poland

2 Department of Psychology and Cognitive Science, Adam Mickiewicz University, Poznan, Poland 


\section{Introduction}

The Theory of Basic Human Values (Schwartz, 1992) postulates that personal values are related to well-being (Sagiv \& Schwartz, 2000). The relationship between values and well-being is strong and straightforward. What individuals consider important and worth pursuing in life is likely to determine the outcomes in their lives. In turn, whether or not individuals perceive that the outcomes in their life meet their expectations shapes their well-being (Kim-Prieto et al., 2005). However, less is known about factors moderating the link between values and well-being. One possible explanation pertains to the role of valuerelated behavior. Research on the behavioral expression of values and their relationship with well-being is relatively sparse and might benefit from a more integrative approach.

The idea that well-being is at its highest when people recognize their values (self-discovery) and are effective in their realization (self-expression) has been emphasized by classical Greek philosophers (see Waterman, 1990a, 1990b), classical psychologists (Maslow, 1954; Rogers, 1951) and modern personality theorists (Schwartz \& Sortheix, 2018; Sheldon \& Krieger, 2014). Moreover, acting upon values is also recognized to enhance wellbeing in modern clinical practices, such as Acceptance and Commitment Therapy (Hayes et al., 1999) and positive psychological interventions (Seligman et al., 2005). For instance, values might provide synergistic effects of value-related beliefs and behavior, e.g., when individuals value others' well-being (Park et al., 2004) and translate their self-transcending beliefs into prosocial actions (Curry et al., 2018).

However, more recent research indicates that some value-related beliefs might result in positive consequences for well-being but have adverse consequences when individuals act upon them. For instance, individuals with conservative beliefs are usually happier than less conservative individuals (Pienaar et al., 2006) because conservative beliefs (e.g., valuing security, conformity, and tradition) provide a basis for higher self-esteem (Van Hiel \& Brebels, 2011). Nevertheless, some instances of conservative behaviors or activism might produce adverse life consequences, e.g., when individuals generate conflict with the outgroup (Jost \& Hunyady, 2002; Schwartz, 2007). This negative feedback loop might be one reason why political activism is less likely among individuals who value conservation than among individuals who value self-transcendence and openness to change (Vecchione et al., 2015).

In the current study, we aimed to examine how the behavioral expression of values relates to well-being. First, we focused on whether value-related behavior is directly associated with well-being outcomes after controlling for value-related beliefs. We also tested whether the link between specific values and well-being depends on whether valuerelated actions accompany beliefs. Finally, whereas studies on values, behavior, and wellbeing have tended to focus on subjective well-being, we chose to extend this investigation to eudaimonic well-being. This perspective is essential in determining the interplay between value-related beliefs and behavior in their relationship with well-being in its broad spectrum.

\subsection{The Structure and Function of Human Values}

Values pertain to what individuals consider important and worth pursuing in life (Schwartz \& Sortheix, 2018; Schwartz et al., 2012). Personal values are motivational constructs that guide intentional human behavior. Individuals are intrinsically rewarded for thoughts and actions congruent with their values and intrinsically punished for incongruent thoughts and 
actions (Feather, 1996; Lewin, 1952; Rohan, 2000; Schwartz, 1992). Values are interconnected so that some of them complement each other (e.g., conformity and tradition) while others stand in opposition to each other (e.g., seeking stimulation vs. security). Therefore, the structure of values takes a circular form. The low angular difference represents values similar to each other, i.e., values that can be realized simultaneously because their underlying motivations are similar. Conversely, values that lie on the opposite parts of the circle cannot usually be realized through a single activity or similar activities because they motivate opposing behaviors. Values form higher-order groups, such as openness to change (self-direction, stimulation, hedonism), self-enhancement (power, achievement), conservation (conformity, tradition), and self-transcendence (benevolence, universalism) (Schwartz et al., 2012). It is debated whether hedonism is best attributed to openness to change or self-enhancement (Lee et al., 2019). However, there seems to be converging empirical evidence that hedonism is more related to openness to change than to self-enhancement (Cieciuch et al., 2014; Giménez \& Tamajón, 2019).

In terms of the values' function, some values are considered healthy because they promote higher subjective well-being, while others hamper it and are considered unhealthy (Sortheix \& Schwartz, 2017). Theorists suggest that healthy values reflect growth and focus on the self. In contrast, unhealthy values reflect deficiency and anxious self-protection (rather than growth) and subordination of the self to social expectations (rather than focusing on the self and personal needs). Thus, openness to change is considered the healthiest value because it motivates individuals to pursue new opportunities that satisfy their personal needs. Individuals who are open to change are expected to achieve greater life satisfaction via self-actualizing, free expression of their ideas, abilities, and feelings. In contrast, conservation is considered the most unhealthy value because it motivates individuals to neglect their own needs for self-actualization and satisfy their ingroup needs for protection and anxiety reduction. Furthermore, self-transcendence and self-enhancement are considered mixed for subjective well-being. Individuals who value self-transcendence are focused on the growth of others rather than their own. Thus, they are likely to contribute to the wellbeing of others more than to their own. Individuals who value self-enhancement are focused on their own needs, which is considered healthy. Nevertheless, individuals motivated by self-enhancement may tend to focus on remedying their personal deficits and miss growth opportunities. For instance, they might be more likely to assert control and dominance over others, e.g., to reduce their anxiety by building self-aggrandizement, thus missing the opportunity to build satisfying social relationships based on mutual benefits.

\subsection{Value-Related Beliefs and Subjective Well-Being}

Research on values aims to explain why personal values influence well-being. For instance, values are expected to promote well-being directly when a value is linked to positive perceptions (e.g., other people are kind), attitudes (e.g., tolerance) and behaviors (e.g., helping), and weaken well-being when a value is linked to negative perceptions (e.g., other people are hostile), attitudes (e.g., prejudice) and behaviors (e.g., shunning others) (Sagiv $\&$ Schwartz, 2000). These direct consequences of values are likely to generate positive or negative emotions and events and, in turn, favorable or unfavorable generalized beliefs about life.

Large-scale studies that used European Social Survey data showed that valuing openness to change and self-transcendence were positively related to life satisfaction and favorable affect balance (Bobowik et al., 2011; Sortheix \& Schwartz, 2017). Self-enhancement 
and conservation were related to lower life satisfaction and a more negative affect balance (Bobowik et al., 2011; Sortheix \& Schwartz, 2017). However, several studies suggested that individuals valuing self-enhancement were more satisfied with life and experienced more positive affect than individuals who did not value achievements (Joshanloo \& Ghaedi, 2009; Oishi et al., 1999; Sortheix \& Lonnqvist, 2015). Moreover, another study found a positive relationship between valuing conservation and satisfaction with life (Karabati \& Cemalcilar, 2010).

Results of these studies show that there is substantial inconsistency in the effects. Broader contexts in which individuals pursue their values can explain this inconsistency, e.g., daily life context or culture. For instance, positive relationships between valuing selfenhancement and life satisfaction and positive affect occurred among business students, but negative relationships occurred for psychology students (Sagiv \& Schwartz, 2000). Cultural factors are also likely to play a role because a study conducted in relatively conservative Turkey presented a positive relationship between conservation and life satisfaction (Karabati \& Cemalcilar, 2010). Finally, one study found a positive relationship between self-enhancement and life satisfaction in developing countries and an inverse relationship in developed countries (Sortheix \& Lonnqvist, 2014).

\subsection{Value-Related Beliefs and Eudaimonic Well-Being}

Well-being is best conceptualized as a compound of hedonia and eudaimonia. Despite research and theory emphasizing a strong link between hedonic and eudaimonic pursuits (Kashdan et al., 2008), these two groups of well-being facets are essential to cover potentially unique effects related to value-oriented action. First, hedonic well-being is often represented by the subjective well-being theory that accounts for cognitive and affective components (Diener, 2000). The cognitive component pertains to the satisfaction with life, i.e., an individual's general belief about his or her life as similar to or different from their subjective ideal. The affective component emphasizes the abundance of positive emotions and the absence of negative emotions in daily life. Second, eudaimonic well-being represents the realization and expression of one's human and individual potential towards personal excellence (Huta \& Ryan, 2010; Kaczmarek, 2017a, b; Waterman, 1990a). This perspective focuses on developing a person's most vital skills and their application to fulfill personally expressed, self-concordant goals. Whereas hedonists primarily seek to feel in a particular way, eudaimonistic individuals seek to be a particular type of person (Huta \& Ryan, 2010; Kaczmarek, 2017a, b).

Considerable attention has been devoted to hedonic well-being in the literature regarding the Theory of Basic Human Values (Schwartz \& Sortheix, 2018), but much less is known about how personal values and their behavioral expression are related to eudaimonic well-being. This is surprising, given that eudaimonia's core lies in identifying and pursuing personally valued goals that contribute to self-expression and self-actualization (Waterman, 1990a). Thus, it might be of central interest to the value theory. Moreover, little is known whether value-related beliefs and behaviors have a different impact on hedonic and eudaimonic well-being. This might be the case because hedonic activities are likely to produce more positive affect (especially in the short-term perspective) and less negative affect (Huta \& Ryan, 2010), but they can be irrelevant to self-actualization. Eudaimonic activities provide more immediate meaning but also decrease the negative affect (Huta $\&$ Ryan, 2010). 
Empirical literature regarding values and eudaimonic well-being is sparse and mostly focuses on beliefs rather than behavior. Research with Polish participants found that valuing openness to change, self-transcendence and conservation were related to higher eudaimonic well-being, while self-enhancement was related to lower eudaimonic well-being (Bojanowska \& Piotrowski, 2017). A positive relationship was also found for conservation and eudaimonic well-being in an Iranian sample (Joshanloo \& Ghaedi, 2009). Finally, Israeli police officers who valued power (self-enhancement) or tradition (conservation) had lower eudaimonic well-being than those who did not endorse these values (Cohen \& Shamai, 2009). In contrast, officers who valued benevolence (self-transcendence), self-direction (openness to change), and achievement (self-enhancement) had higher eudaimonic well-being than those who valued them less. Consequently, the literature suggests that openness to change and self-transcendence are related to higher eudaimonic well-being, conservation effects are mixed (but relatively positive), and self-enhancement effects are also mixed (and rather negative).

However, there might be a cultural variation as the results were different in Israel, where the culture is centered around self-enhancement than in Poland, centered around conservation (Schwartz, 2007). According to Hofstede's (2018) six cultural dimensions, Polish society is rather traditional, hierarchical, individualistic, and masculine, with substantial uncertainty avoidance, normative orientation, and high restraint. Thus, in the present study, we expected to find a positive relationship between eudaimonic well-being and conservation because person-environment value congruence is beneficial for well-being (Sortheix \& Lönnqvist, 2015). Moreover, we also expected a positive relationship between eudaimonic well-being and valuing openness to change and self-transcendence, and a negative relationship with self-enhancement.

\subsection{Value-Related Behavior and Well-Being}

Achieving value-related goals corresponds with higher subjective well-being for two reasons. First, value-related behaviors (e.g., helping others resulting from self-transcendence) are likely to generate positive events along with positive emotions (experiencing emphatic joy) and cognitions ("I am a good person," "I live in a world where people help each other"). Second, successful value-related actions lead to an intrinsically rewarding experience of goal attainment (Schwartz \& Sortheix, 2018). Valuing a particular goal indicates that the goal was personally meaningful, regardless of whether the action was good or bad from the observers' perspective. This phenomenon has been termed 'walking the talk' (i.e., performing actions consistent with one's claims). Consequently, values enactment might be linked to the well-being more than value-related beliefs (Sheldon \& Krieger, 2014). The value-behavior fit is essential because when internalized values guide behavior, individuals engage in self-concordant actions that promote well-being. For instance, self-concordant work facilitates well-being (Henricksen \& Stephens, 2010, 2013). This approach has also been emphasized in the strengths of character perspective, in which initiating actions that reflect moral traits led to behaviors that increase well-being (Schutte \& Malouff, 2019).

Some authors have questioned the robustness of the link between what people think is worth pursuing in life and what they actually do, advocating for human moral hypocrisy (e.g., Batson \& Thompson, 2001). Nonetheless, research indicated that value-related beliefs correlate with behavior (Bardi \& Schwartz, 2003; Schwartz \& Butenko, 2014). There is variation in how value-related beliefs and behaviors relate to each other. For instance, values and behaviors correlate more strongly for stimulation (openness to change component) 
and tradition (conservation) than for security and conformity (conservation), achievement (self-enhancement), and benevolence (Self-transcendence) (Bardi \& Schwartz, 2003). These findings suggest that value-related beliefs and behaviors are interrelated but distinct constructs. Bardi and Schwartz (2003) suggest that the belief-behavior link is not straightforward when individuals are under normative social pressure. This is another reason why a culture that provides external normative pressures is likely to diminish the role of personal beliefs in explaining behavior (Roccas \& Sagiv, 2010). Thus, studying the links and influences of value-related beliefs and behavior is essential in diverse cultural contexts.

Finally, studying value-related beliefs and behaviors as separate constructs is essential because some values might have a different impact on well-being depending on the frequency with which individuals act upon them. For instance, individuals who value conservation are likely to protect the self, resulting in higher self-esteem (Van Hiel \& Brebels, 2011) and less distress (Van Hiel \& De Clercq, 2009). Conservative attitudes are likely to become problematic when they protect the self but become harmful to others, e.g., outgroups such as immigrants (Jost \& Hunyady, 2002; Schwartz, 2007). Thus, valuing conservation can provide more well-being among passive individuals who use conservative beliefs to enhance their meaning in life and remedy anxieties. These findings are important from the cultural perspective because Poles are among the nations with the highest conservation indices (Schwartz, 2007).

For self-enhancement, cherishing personal attributes of power (valuing power) might compensate for a sense of low self-worth for some individuals. Thus, individuals who value power might experience greater well-being, but their beliefs may also translate into socially unhealthy behavior, e.g., rivalry rather than cooperation. Power-related behavior is likely to generate interpersonal conflict or criticism once individuals attempt to act upon their power, e.g., enforcing rather than assertively negotiating specific responses from others (Sarkova et al., 2013). In such scenarios, individuals might experience higher eudaimonic well-being (greater personal development and self-expression), but lower subjective well-being (more negative emotions and lower life satisfaction) when they initiate these value-related actions. For instance, a satisfying feeling of accomplishment (e.g., achieving better work results than colleagues related to the achievement value; or successfully forcing someone to yield, related to the power value) might mix with dissatisfaction in terms of adverse social outcomes (e.g., more interpersonal conflict). Consequently, individuals might derive more benefits for their subjective well-being from their self-enhancement beliefs (e.g., experiencing power via self-aggrandizement) than from actual behaviors.

Openness to change beliefs and behaviors might also produce different outcomes for well-being. For instance, individuals who consider themselves hedonists (high valuing of pleasure) might experience higher subjective well-being because they maintain positive beliefs, i.e., focus on, think, and know more about positive aspects of life. Moreover, hedonists may also initiate actions (hedonism-related activities) that promote positive hedonic outcomes (e.g., engaging in more enjoyable leisure activities) (Giuntoli et al., 2020; Huta \& Ryan, 2010). Such behaviors might have relatively little impact on eudaimonic well-being. Hedonistic pursuits are usually irrelevant to self-actualization as they mostly influence what people experience rather than what people are. Moreover, hedonism can backfire in the long run if individuals initiate risky behaviors or postpone obligations for the sake of fun.

In contrast, benevolent individuals (self-transcendence) derive satisfaction from favorable beliefs about the world and the self (Fredrickson et al., 2008) and derive even more wellbeing from benevolent actions (Curry et al., 2018). For instance, individuals characterized by high trait kindness (Park et al., 2004), those who increase their kindness via meditation 
(Fredrickson et al., 2008) or perform kind acts (Curry et al., 2018) achieve greater well-being. Nonetheless, there are concerns about whether individuals excessively focused on self-transcendence contribute to others' well-being at the cost of impeding their own subjective wellbeing (Bartlett \& DeSteno, 2006; Sortheix \& Schwartz, 2017). For instance, individuals might deprive themselves of vital resources by transferring them to others. This can impede subjective well-being when highly self-transcending individuals act upon their values in a social surrounding that is low in self-transcendence and is not likely to compensate the self-transcending individual for their prosocial contribution.

In conclusion, there is a theoretical and empirical rationale for studying the impact of value-related beliefs and behavior. These two aspects of values are related, but they can have a different impacts on subjective and eudaimonic well-being.

\section{Present Study}

Building upon the Theory of Basic Human Values (Sagiv \& Schwartz, 2000; Schwartz, 1992), we aimed to examine the relationships between value-related beliefs, value-related behavior, and well-being outcomes. First, we sought to examine the role of value-related behavior in predicting well-being above the value-related beliefs. For instance, we aimed to test whether individuals who hold specific values are happier than individuals who do not hold these values and whether they are happier when they act upon their values than individuals who remain passive.

Second, we focused on moderations in which value-related beliefs and behaviors interact with each other in their relationship with an individual's well-being. Testing for moderation provides information about conditional effects, i.e., whether values are associated with higher (or lower) well-being on the condition that relevant actions accompany them. Some values might be related to higher well-being on the condition that they are accompanied by (enhancement) or are not accompanied by (suppression) congruent actions. The link between beliefs and well-being might be suppressed or enhanced depending on whether individuals see the positive or negative influence of their value-related behavior on well-being. For instance, individuals might derive less satisfaction from their power-related beliefs if their social environment is resistant to their influences.

We included eudaimonic and hedonic measures to cover two aspects of human well-being. First, we conceptualized eudaimonic well-being building upon Waterman's framework, which introduces classical philosophers' ideas into psychology (Waterman, 1990a, b). Second, we conceptualized hedonic well-being following Diener's subjective well-being approach (Diener, 2000; Kim-Prieto et al., 2005). Individuals have high subjective well-being when they experience an abundance of positive emotions and low levels of negative emotions, and evaluate their life as positive: a combination that corresponds with classical hedonism notions (Huta \& Waterman, 2014; Kaczmarek, 2017a). We used two facets of well-being to cover its spectrum. Given the current state-of-the-art, we explored whether the relationships between values and specific facets of well-being exist, rather than predicting the exact directions of relationships. 


\section{Method}

\subsection{Participants}

Participants were 1,161 Polish adults (55\% women) aged between 18 and 78 years $(M=45$, $S D=15.01)$. The study was conducted online in Polish via a professional research panel Ariadna. We collected data from a national sample representative of gender, age, education, and Poland's regions. We included two control questions (e.g., "In this item, chose answer 4") and excluded data with extremely short response times or no variation between answers. In this way, we removed $15 \%$ of the initial data. After examining the removed participants' demographics, we then collected additional data to compensate for these losses. Each participant provided written informed consent. An Ethics Committee approved the study.

\subsection{Measures}

\subsubsection{Well-Being}

3.2.1.1 Eudaimonic Well-Being We used the Questionnaire for Eudaimonic Well-being (Waterman et al., 2010; adapted into Polish by Kłym et al., 2014). It consists of 21 items (e.g., 'I believe I know what my strongest skills are and I try to develop them whenever possible'), with answers ranging from 1 ('strongly agree') to 7 ('strongly disagree'). Higher scores indicate higher eudaimonic well-being. This scale had satisfactory reliability with Cronbach's $\alpha=0.88$.

3.2.1.2 Positive and Negative Affect Positive affect and negative affect were measured using the Positive and Negative Affect Schedule (Bojanowska \& Zalewska, 2015; Watson et al., 1988). This measure comprises a list of ten adjectives referring to positive (e.g., interested, excited) and ten adjectives referring to negative (e.g., guilty, ashamed) affective states experienced over the previous two weeks. Participants respond on a scale of 1 ('slightly or not at all') to 5 ('extremely'). Higher scores represent higher intensity of affect. The scales showed satisfactory reliability with Cronbach's $\alpha=0.86$ for positive affect and $\alpha=0.91$ for negative affect.

3.2.1.3 Satisfaction with Life We used the Satisfaction with Life Scale (SWLS) (Bojanowska \& Zalewska, 2015; Diener et al., 1985) to assess participants' global evaluation of their life ('In most ways, my life is close to my ideal') on a scale from 1 ('I definitely disagree') to 7 ('I definitely agree'). Higher scores represent higher satisfaction with life. This scale had satisfactory reliability with Cronbach's $\alpha=0.90$.

\subsubsection{Human Values}

To measure what participants valued in their lives according to Schwartz's 19-values model, we used the Portrait of Values Questionnaire (PVQ; Schwartz et al., 2012; adapted into Polish by Cieciuch, 2013). This questionnaire consists of 57 brief descriptions (or portraits) of different individuals, with three descriptions for each value. Each description 
portrays a person's goals and aspirations, introduced with words such as 'It is important to him/her,' 'He/she thinks,' or 'He/she believes.' We grouped the 19 values into four higher-order dimensions. Self-transcendence comprised universalism and benevolence and included descriptions such as 'He goes out of his way to be a dependable and trustworthy friend'. Openness to change comprised hedonism stimulation and self-direction and included descriptions such as 'It is important to him to have a good time'. We ascribed hedonism to openness to change because it correlated with other values from this dimension more strongly (correlations within the range from $r=0.35$ to $r=0.53$ ) compared to values from the self-enhancement dimension (correlations within the range from $r=0.17$ to $r=0.35$ ). Conservation comprised security, conformity, humility and tradition, and included descriptions such as 'It is important to him to be humble.' Finally, self-enhancement comprised power-dominance, power-resources, and achievement, and included descriptions such as 'Being very successful is important to him.' Participants were asked to indicate 'How much like you is this person?' using a six-point scale ranging from 'Very much like me' to 'Not like me at all.' Reliability was satisfactory for all four scales: selfenhancement, $\alpha=0.86$; openness to change, $\alpha=0.85$; conservation, $\alpha=0.90$; and, self-transcendence, $\alpha=0.91$.

\subsubsection{Value-Related Behaviors}

To assess value-related behaviors, we developed a questionnaire in which people reported how often they expressed their values in their actions. Based on Schwartz's model (Schwartz et al., 2012), we created a pool of behaviors for each value and conducted a pilot study to identify items with the best psychometric properties. The pilot sample consisted of 515 university students aged 17 to $61(M=29.05, S D=8.89)$. They filled out the questionnaire indicating how often they behaved in specific ways ( 84 behaviors expressing values) in the previous 12 months. We excluded 27 items with behaviors that correlated weakly with other value-related behaviors from the dimension they represented. The scale structure was consistent with the 19 values structure, with factor weights supporting the circumplex structure, and with the model of four higher-order dimensions of values, with RMSEA below 0.08 and CFI 0.90 respectively (Schreiber et al., 2006), showing satisfactory fit. Values correlated strongest with their respective value-expressive behaviors. This was true both for the 19 values and for the four higher-order dimensions. The final version of the questionnaire comprised three behaviors for each value (e.g., 'I kept promises made to my family or friends' for benevolence). Respondents indicated how often they behaved this way when they had the opportunity. They used a scale ranging from 0 ('never') to 4 ('always'). Higher scores expressed higher engagement in particular behaviors. The reliability of the scales in the present study was satisfactory for all four value-related behaviors: self-enhancement, $\alpha=0.85$; openness to change, $\alpha=0.75$; conservation, $\alpha=0.82$; and, self-transcendence, $\alpha=0.88$.

\subsubsection{Analytical Strategy}

To test our hypotheses, we conducted a series of regression analyses for each well-being component and each value dimension using Hayes PROCESS macro (model 1, simple moderation; Hayes, 2013). We introduced well-being indices as the outcome, values as the predictor, behaviors as the moderator, and gender and age as covariates. Thus, the models tested whether (controlling for age and gender) individuals who endorsed a specific value 
were more likely to be happier on the condition that they performed actions relating to that value. For greater clarity, we have not presented the coefficients for gender and age because they were not the subject of our hypotheses (Table 2). To control for family-wise error, we corrected each $p$-value for False Discovery Rate for the hypothesized outcomes (Benjamini \& Hochberg, 1995).

\section{Results}

Table 1 presents descriptive statistics and correlations.

\subsection{Self-enhancement}

We found that valuing self-enhancement related to higher eudaimonic well-being and positive affect (Table 2). However, individuals who initiated more self-enhancement actions had lower eudaimonic well-being, higher negative affect, and higher positive affect.

\subsection{Openness to Change}

Individuals who valued openness to change had higher well-being, i.e., higher eudaimonic well-being, satisfaction with life and positive affect, and lower negative affect (Table 2). More frequent behaviors related to openness to change correlated with higher satisfaction with life, higher positive affect, and higher negative affect. We observed that behavior moderated the inverse relationship between beliefs and negative affect. The relationship was stronger for individuals who initiated more value-related actions than those who remained passive (Fig. 1).

\subsection{Conservation}

Valuing conservation was related to higher eudaimonic well-being, satisfaction with life, positive affect, and lower negative affect. Conservation behavior was not directly related to any well-being measure. However, behavior moderated the link between value-related beliefs and well-being. The link between conservation beliefs and eudaimonic well-being was stronger for passive individuals than for active individuals (Fig. 2). In contrast, the link between conservation beliefs and positive affect was stronger for active individuals than for passive individuals (Fig. 3).

\subsection{Self-transcendence}

Valuing self-transcendence was related to higher eudaimonic well-being, higher positive affect, and lower negative affect but not satisfaction with life. Acting upon self-transcendence was related to higher eudaimonic well-being, satisfaction with life, and positive affect. Furthermore, self-transcending behavior moderated the link between beliefs and eudaimonic well-being, and positive affect. The link between beliefs and eudaimonic well-being (Fig. 4) as well as positive affect (Fig. 5) was stronger for individuals who acted upon their self-transcendence rather than were disengaged. 


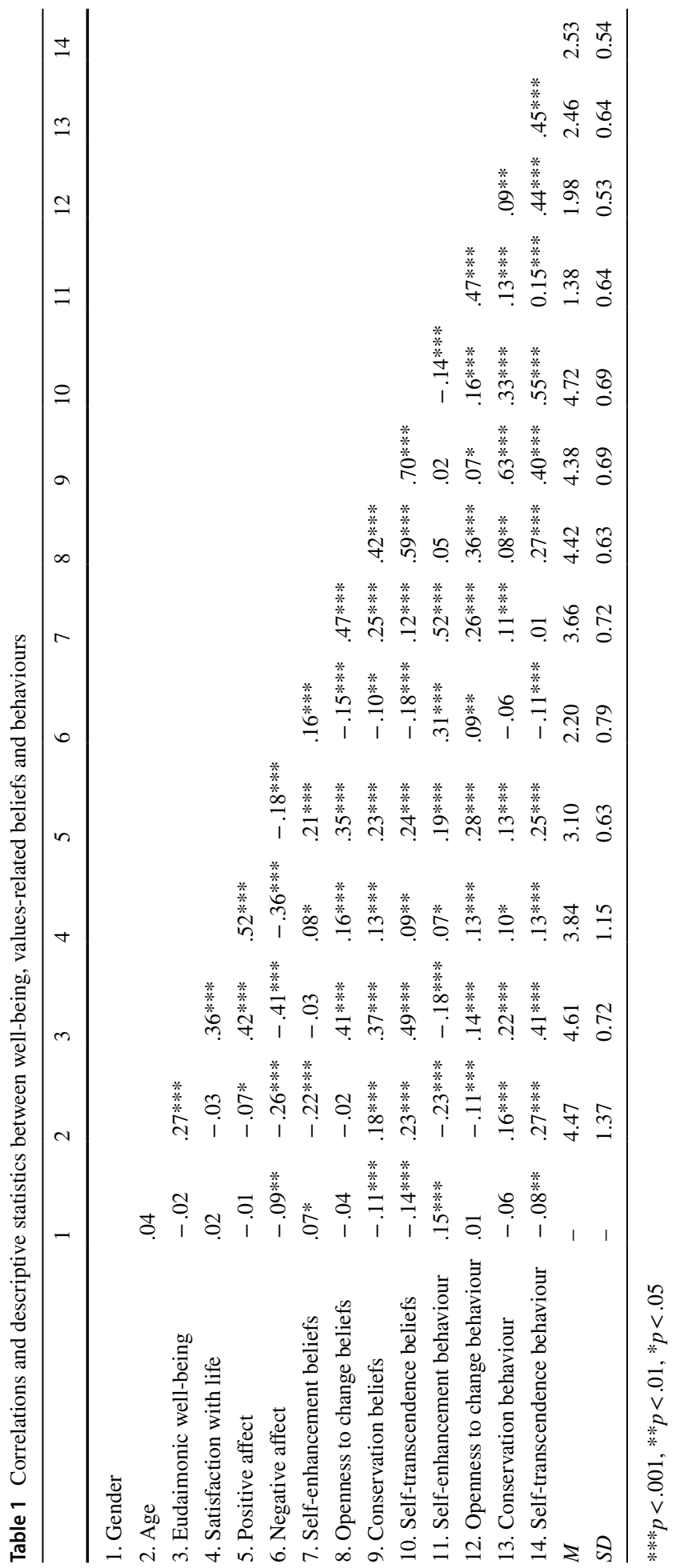


222

A. Bojanowska, Ł. D. Kaczmarek

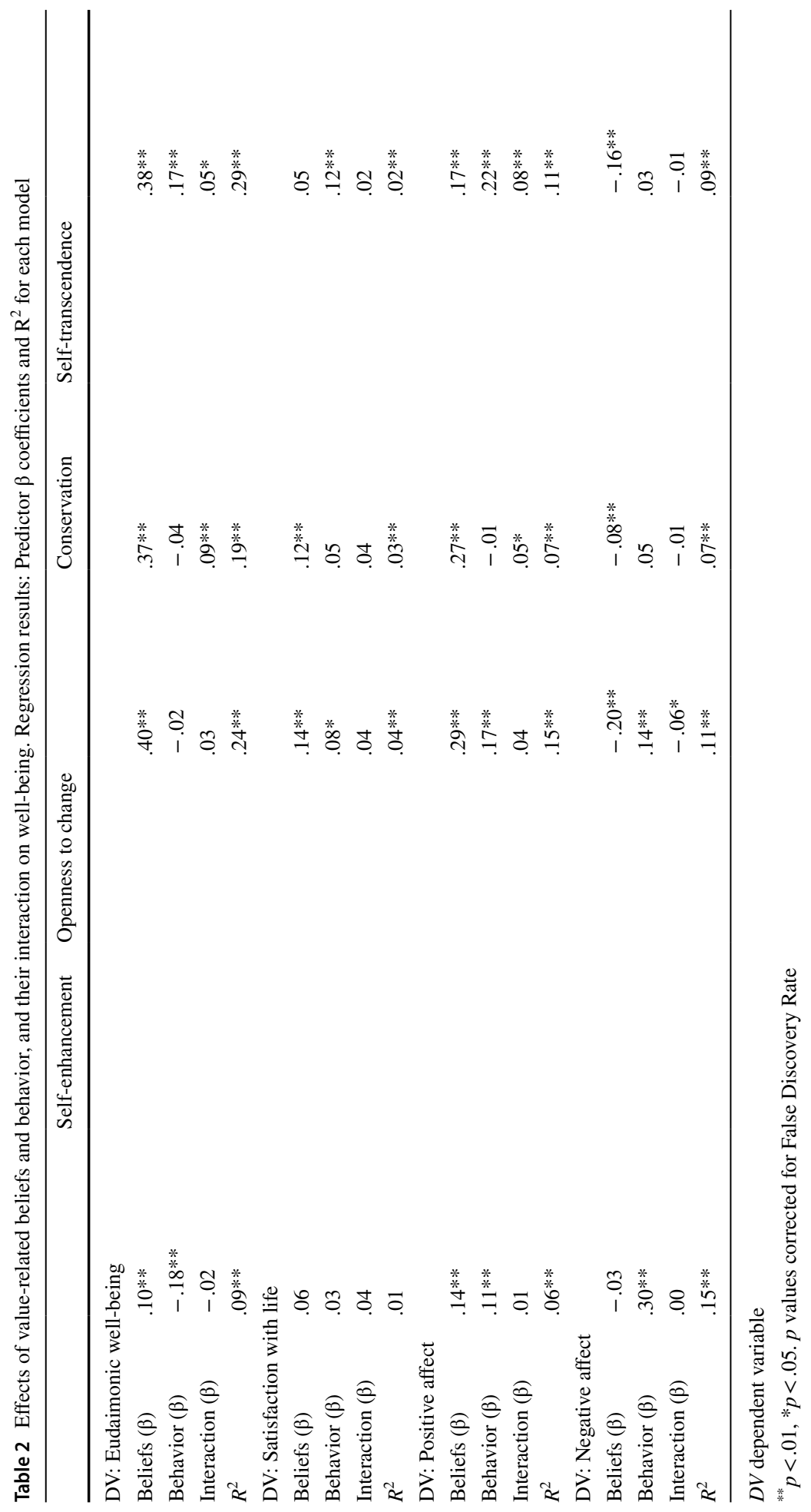

Epringer 


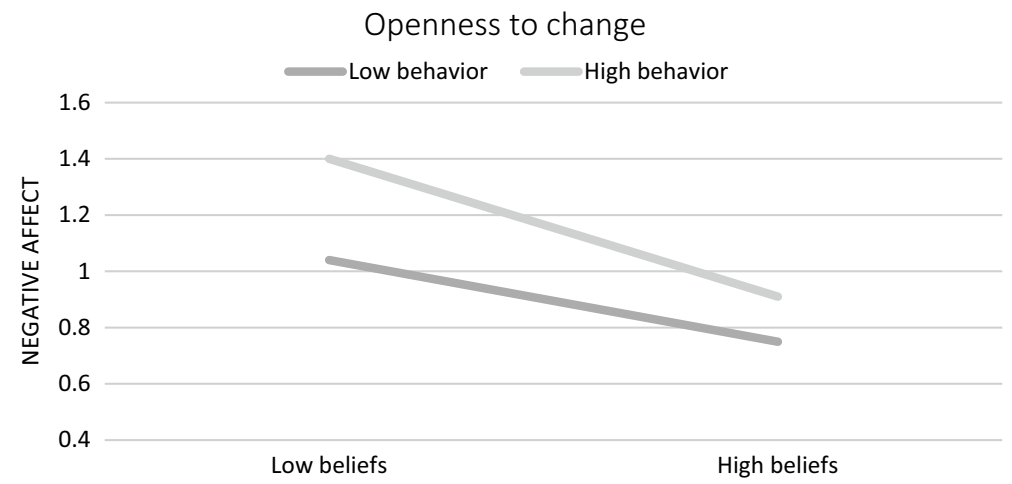

Fig. 1 Interaction of openness to change beliefs and behavior in their relationship with negative affect

\section{Conservation}

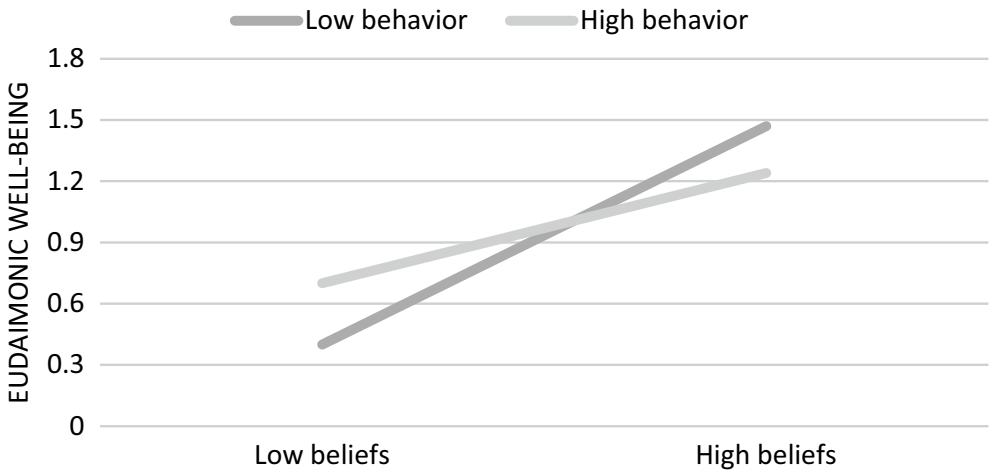

Fig. 2 Interaction of conservation beliefs and behavior in their relationship with eudaimonic well-being

\section{Conservation}

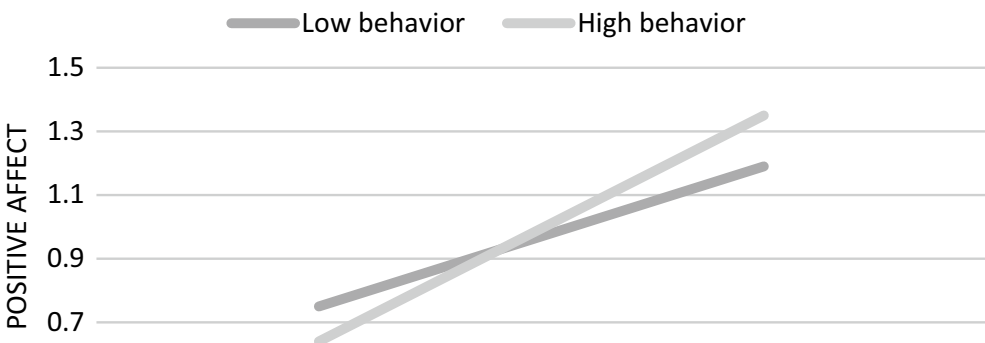

0.5

Low beliefs High beliefs

Fig. 3 Interaction of conservation beliefs with behavior in their relationship with positive affect 


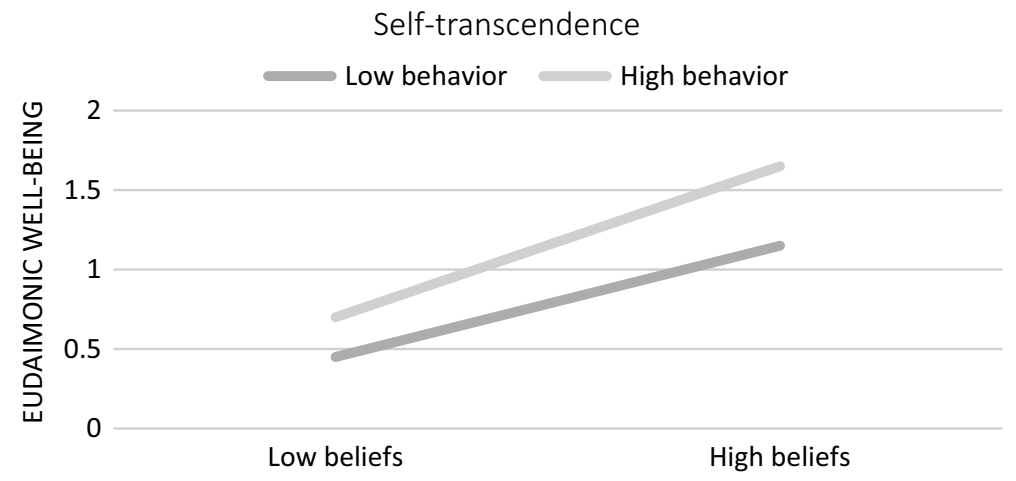

Fig. 4 Interaction of self-transcendence beliefs with behavior in their relationship with eudaimonic wellbeing

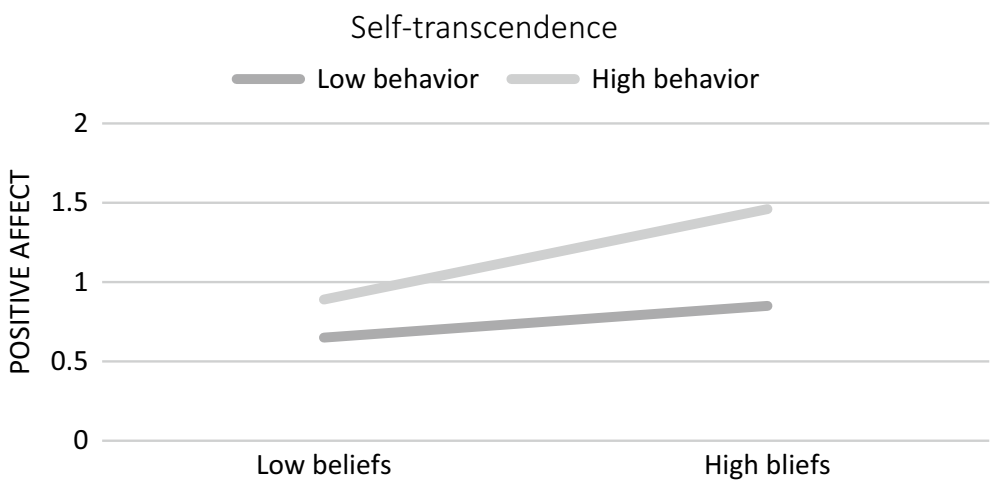

Fig. 5 Interaction of self-transcendence beliefs with behavior in their relationship with positive affect

\section{Discussion}

We examined how values (i.e., beliefs about what is essential in life), value-related behaviors (actual actions aimed at enhancing valued aspects of life), and their interaction are related to hedonic and eudaimonic components of well-being. Our results contribute to a more nuanced understanding of how values are linked to meaningful life outcomes (Schwartz \& Sortheix, 2018). Overall, we found that specific values were related to wellbeing and that value-related behaviors were additional, independent predictors of wellbeing indices in several cases. For some values and aspects of well-being, behavior moderated the link between beliefs and well-being. Consequently, for self-transcendence, this link was stronger when individuals initiated many value-related actions. For self-enhancement and conservation, the link was stronger for more passive individuals. This means that accounting for value-related behavior provides additional information on whether a person's well-being is best explained by value-related beliefs or rather some other factors. It is consistent with the concept of intrinsic motivation in Self-Determination Theory (Ryan \& Deci, 2000) and Waterman's definition of eudaimonic well-being as the expression of one's true self (Waterman et al., 2010). 
We found support for the hypothesis that some values are healthy because acting upon these values is related to greater well-being, some values are unhealthy because acting upon them is related to lower well-being, and some are mixed (Sagiv \& Schwartz, 2000). In our study, self-transcendence had the most consistent positive relationships with components of well-being. In contrast, self-enhancement can be considered a mixed value from the perspective of well-being. Individuals who focused on self-enhancement experienced higher eudaimonic well-being, while individuals who engaged in self-enhancing behavior had lower eudaimonic well-being. This supports the claim that building life projects on values related to remedying deficits is a pursuit that corresponds with lower well-being.

This project revealed more detailed information on how specific values (beliefs, actions, and their interaction) contribute to four facets of well-being. First, we found that individuals who endorsed strong self-enhancement beliefs (valuing power and achievements) experienced more eudaimonic well-being and positive affect. However, individuals who initiated more self-enhancement practices (regardless of whether they valued these activities or not) experienced less eudaimonic well-being and more negative affect; and somewhat more positive affect. No interaction occurred, suggesting that the effects of self-enhancement beliefs on well-being were not conditional upon behavior. As noted above, these findings indicate that self-enhancement is a value that is best kept passive. Individuals focused on increasing power and collecting achievements might benefit from more consideration of how their actions influence others and whether there are ways in which these individuals' urges to increase power might be adapted to fit the expectations of their social surroundings.

Self-enhancement action was related to higher levels of negative and positive affect. This suggests that self-enhancement behavior was associated with higher negative and positive emotions rather than either negative or positive emotions. Thus, individuals who initiate self-enhancement behavior more often are characterized by mixed emotions (Larsen \& McGraw, 2011). This finding might be relevant to the study of emodiversity; a theory that emphasizes the benefits of experiencing a wide range of positive and negative emotions in life (Quoidbach et al., 2014). High emodiversity is believed to promote social and physical health (Grossmann et al., 2019). However, we found evidence for the co-occurrence of higher positive and negative affect in individuals whose behavior was detrimental to their eudaimonic well-being. This might indicate that emodiversity is not uniformly related to positive outcomes. Thus, future studies might focus on emodiversity in the context of emotional aspects of self-enhancement.

We observed that individuals who valued openness to change (i.e., hedonism, stimulation, and self-direction) had greater eudaimonic well-being, derived more life satisfaction, experienced more positive affect and less negative affect. Moreover, when they initiated behaviors typical for openness to change (e.g., seeking pleasure or stimulating activities), they had higher levels of life satisfaction and positive affect, yet also higher comparable negative affect levels. There was a significant interaction of openness to change beliefs and behavior in their relationship with negative affect. The inverse association between openness to change beliefs and negative affect was stronger for individuals who initiated more openness to change actions. Namely, we observed the highest negative affect levels among individuals who did not value openness to change but exhibited more openness to change behavior. This finding might correspond with the notion that self-concordant actions (e.g., a job that reflects personal interests) promote greater well-being (Henricksen \& Stephens, 2010). Consequently, individuals who initiate more self-discordant actions (activities that they do not consider worth pursuing in life) might experience more negative outcomes such as higher negative affect. Second, the lowest negative affect was observed for individuals 
who valued openness to change but initiated few openness to change actions. This finding is also worthwhile because it indicates that individuals who experienced the lowest negative affect levels maintained specific beliefs (e.g., valuing pleasure, fun, novelty, and excitement) yet did not translate many of these beliefs into action. It suggests that for openness to change and its relationship with negative affect, what individuals think about themselves and the world is more important than what they actually do in their lives.

For conservation (e.g., valuing security, conformity, and tradition), beliefs were related to higher eudaimonic well-being, satisfaction with life, positive affect, and lower negative affect. We observed no independent relationships of conservative behavior with well-being. This finding suggests that for conservation, what individuals believe in is important for well-being, but what they do is not. This finding is in line with previous studies that found greater eudaimonic well-being and satisfaction with life (Karabati \& Cemalcilar, 2010; Pienaar et al., 2006), higher self-esteem (Van Hiel \& Brebels, 2011), and less distress (Van Hiel \& De Clercq, 2009) among more conservative individuals. However, this finding is not in line with some other studies that found lower positive affect among individuals who valued conservation (Sagiv \& Schwartz, 2000). It also does not support theoretical considerations that related conservation to the lowest levels of subjective well-being due to subordinating the self to socially imposed expectations and a protective or anxiety-rather than growth-oriented focus (Sortheix \& Schwartz, 2017).

Conservation-related behavior interacted with beliefs in their association with eudaimonic well-being and positive affect. For conservation, the relationship between values and eudaimonic well-being was stronger for passive individuals, i.e., those who initiated fewer actions. We observed the highest eudaimonic well-being among individuals who endorsed conservation values but initiated fewer conservation-related actions. We observed the lowest eudaimonic well-being among individuals who did not endorse conservation values and displayed poor conservation-related behavior. In contrast, the relationship between values and positive affect was stronger for active individuals. These findings are significant because they indicate that conservation-related behavior was not directly associated with well-being but was a significant moderator of values-related beliefs and well-being. For conservation, cognition (what individuals perceive as important in life) seems to be more related to well-being than actual behavior. It is in line with some previous studies regarding conservatism's adverse effects (Jost \& Hunyady, 2002; Schwartz, 2007). However, conservation-related beliefs were linked more strongly with positive affect when individuals remained active. Thus, taken together, conservation-related activity seems to boost the emotional but suppress the eudaimonic consequences of conservation. In contrast, more passive individuals derived more eudaimonic yet less emotional benefits from their conservative beliefs. To conclude, these results indicate that whether individuals who value conservation remain active versus passive may have a differential effect on specific aspects of well-being. These findings are worthwhile from the cultural perspective because Poles are among the nations with the highest conservation (Schwartz, 2007). Thus, they present how conservation operates in a conservative culture.

We found that among individuals who endorsed self-transcendence (recognition of benevolence and universalism), stronger beliefs were associated with higher eudaimonic well-being and positive affect and lower negative affect. It indicates that individuals who appreciated the role of self-transcendence in life were also happier, i.e., they were more effective in expressing their true self (Waterman et al., 2010) and experienced more positive emotions. Moreover, individuals who initiated more self-transcendence actions had higher eudaimonic well-being, satisfaction with life, and positive affect. Thus, an active and self-concordant approach towards self-transcendence characterized the individuals 
that were happier than their more passive counterparts and individuals who rejected selftranscendence as a vital aspect of life. These findings are consistent with previous studies that advocated for self-transcendence benefits (Bobowik et al., 2011; Bojanowska \& Piotrowski, 2017). However, we found no support for the expectation that self-transcendence might also be detrimental to well-being as individuals invest more in others than in themselves (Sortheix \& Schwartz, 2017). We found the opposite, self-transcending behavior produced more eudaimonic well-being (self-expression and self-actualization), as well as more hedonic well-being (more positive emotions and life satisfaction and less negative affect). This supports theories that emphasize the mutual benefits of altruistic or prosocial behavior for the giver and the taker (Curry et al., 2018; Hui et al., 2020).

We also observed that value-related actions moderated the relationship of self-transcendence beliefs with eudaimonic well-being and positive affect. The association between valuing self-transcendence and well-being (eudaimonic well-being and positive affect) was more robust for active individuals, i.e., those engaged in many activities, relative to disengaged individuals. The link between self-transcendence beliefs and eudaimonic wellbeing and self-transcendence beliefs and positive affect was stronger for individuals who initiated more self-transcending acts than those who initiated few Self-transcending acts. Thus, for self-transcendence, the moderating role of behavior was straightforward, unlike for self-enhancement and conservation. Our results indicate that self-transcendence beliefs and actions had independent and positive associations with several well-being components; a finding that supports consideration of self-transcendence as a healthy rather than mixed value (Sortheix \& Schwartz, 2017).

Finally, we also found evidence that values, value-related actions, and values conditional on action had a differential correspondence to hedonic well-being and eudaimonic well-being. For instance, self-enhancement related to higher well-being, although individuals who initiated actions relating to these values experienced greater negative affect (we found no effect for life satisfaction). This contradicts previous arguments for streamlining the different well-being components into a more general factor (Disabato et al., 2016). Perhaps, high structural correlations between different well-being components matter less when functional differences are analyzed, as it was in our study.

This study has several limitations. First, although regression analysis allows for the interpretation of causal effects (Pearl, 2012), only an experimental design that manipulated beliefs and behaviors relating to values could provide direct evidence of the causal validity of our conclusions. In this regard, future studies might test how enhancing or inhibiting certain beliefs or behaviors via interventions influences well-being outcomes. More experimental evidence is essential to guide evidence-based interventions that participants could receive in clinical settings. This is particularly important, given that more recent evidence indicates that the current well-being interventions produce weaker effects than those reported in earlier studies (Cregg \& Cheavens, 2020). Moreover, longitudinal designs would also be more suitable for explaining how changes in values-related beliefs and behavior predict well-being changes. Second, we focused on retrospective self-reports of behavior. Although self-reports of behavior are usually relatively accurate in terms of rank order (e.g., Johns \& Miraglia, 2015), more direct measuring behavior methods could corroborate our findings. Third, as we report several findings that we explored but did not hypothesize, replication is needed to ascertain that these findings are robust. Finally, we did not account for the congruence between individuals' values and prevailing values in society, proposed as an additional explanatory mechanism linking values and well-being (Schwartz \& Sortheix, 2018). Future studies might usefully consider this additional factor, e.g., by incorporating a cross-cultural perspective and addressing social norms explicitly. 
This study has several practical implications. First, our findings emphasize the benefit of asking about beliefs, behaviors, and their interaction when explaining well-being levels. Thus, interventionists might use this knowledge to develop more nuanced interviewing, assessment, and psychological help. It is also imperative to address that self-enhancement values are unhealthy when they translate into specific self-enhancing behaviors. In this case, people might lose their basis for well-being once they turn their beliefs into intense actions. This evidence should warn psychologists or coaches when helping their clients achieve their goals; they should make them aware that some valued aims could pose a risk to their well-being. It is crucial given that we observed self-enhancement beliefs to produce higher well-being when analyzed separately. Finally, our findings may be relevant to understanding social or political activism resulting from valuing self-transcendence and openness to change (Vecchione et al., 2015). Investigations that dissect value-related beliefs from behavior are likely to explain difficulties that some individuals might experience while becoming more active, e.g., by becoming social or political activists.

In summary, these findings provide new evidence of the benefits of separating values from actions and recognizing their interaction when predicting and regulating well-being. Moreover, our results present the benefits of distinguishing between hedonic and eudaimonic components of well-being in their relationship with values. Some of our findings did not support the theoretical predictions regarding the link between values and wellbeing. This suggests that the Theory of Basic Human Values might need further functional refinement.

Data Availability Data link: https://www.researchgate.net/publication/340398876_values_wellbeing.

\section{Declarations}

Conflict of interest The authors declare that they have no conflict of interest.

Ethical Approval The study was approved by the SWPS University Ethical Committee.

Informed Consent All participants provided informed consent.

Open Access This article is licensed under a Creative Commons Attribution 4.0 International License, which permits use, sharing, adaptation, distribution and reproduction in any medium or format, as long as you give appropriate credit to the original author(s) and the source, provide a link to the Creative Commons licence, and indicate if changes were made. The images or other third party material in this article are included in the article's Creative Commons licence, unless indicated otherwise in a credit line to the material. If material is not included in the article's Creative Commons licence and your intended use is not permitted by statutory regulation or exceeds the permitted use, you will need to obtain permission directly from the copyright holder. To view a copy of this licence, visit http://creativecommons.org/licenses/by/4.0/.

\section{References}

Bardi, A., \& Schwartz, S. H. (2003). Values and behavior: Strength and structure of relations. Personality and Social Psychology Bulletin, 29(10), 1207-1220. https://doi.org/10.1177/0146167203254602

Bartlett, M. Y., \& DeSteno, D. (2006). Gratitude and prosocial behavior helping when it costs you. Psychological Science, 17, 319-325. https://doi.org/10.1111/j.1467-9280.2006.01705.x

Batson, C. D., \& Thompson, E. R. (2001). Why don't moral people act morally? Motivational considerations. Current Directions in Psychological Science, 10(2), 54-57. https://doi.org/10.1111/1467-8721. 00114 
Benjamini, Y., \& Hochberg, Y. (1995). Controlling the false discovery rate: A practical and powerful approach to multiple testing. Journal of the Royal Statistical Society Series B (Methodological), 57(1), 289-300. https://doi.org/10.2307/2346101

Bobowik, M., Basabe, N., Paez, D., Jimenez, A., \& Bilbao, M. A. (2011). Personal values and well-being among Europeans, Spanish natives, and immigrants to Spain: Does the culture matter? Journal of Happiness Studies, 12, 401-419. https://doi.org/10.1007/s10902-010-9202-1

Bojanowska, A., \& Piotrowski, K. (2017). Values and psychological well-being among adolescents-Are some values "healthier" than others? European Journal of Developmental Psychology, 16(4), 1-15. https://doi.org/10.1080/17405629.2018.1438257

Bojanowska, A., \& Zalewska, A. (2015). Lay understanding of happiness and the experience of well-being: Are some conceptions of happiness more beneficial than others? Journal of Happiness Studies, 17(2), 793-815. https://doi.org/10.1007/s10902-015-9620-1

Cieciuch, J. (2013). Ksztattowanie się systemu wartości od dzieciństwa do wczesnej dorosłości The formation of a value system from childhood to early adulthood. Warszawa: LiberiLibri.

Cieciuch, J., Davidov, E., Vecchione, M., \& Schwartz, S. H. (2014). A hierarchical structure of basic human values in a third-order confirmatory factor analysis. Swiss Journal of Psychology, 73(3), 177-182. https://doi.org/10.1024/1421-0185/a000134

Cohen, A., \& Shamai, O. (2009). The relationship between individual values, psychological well-being, and organizational commitment among Israeli police officers. International Journal of Police Strategies \& Management, 33, 30-51. https://doi.org/10.1108/13639511011020584

Cregg, D. R., \& Cheavens, J. S. (2020). Gratitude interventions: Effective self-help? A meta-analysis of the impact on symptoms of depression and anxiety. Journal of Happiness Studies. https://doi.org/10.1007/ s10902-020-00236-6

Curry, O. S., Rowland, L. A., Van Lissa, C. J., Zlotowitz, S., McAlaney, J., \& Whitehouse, H. (2018). Happy to help? A systematic review and meta-analysis of the effects of performing acts of kindness on the well-being of the actor. Journal of Experimental Social Psychology, 76, 320-329. https://doi.org/ 10.1016/j.jesp.2018.02.014

Diener, E. (2000). Subjective well-being: The science of happiness and a proposal for a national index. American Psychologist, 55(1), 34-43. https://doi.org/10.1037/0003-066X.55.1.34

Diener, E., Emmons, R. A., Larsen, R. J., \& Griffin, S. (1985). The satisfaction with life scale. Journal of Personality Assessment, 49, 71. https://doi.org/10.1207/s15327752jpa4901_13

Disabato, D. J., Goodman, F. R., Kashdan, T. B., Short, J. L., \& Jarden, A. (2016). Different types of wellbeing? A cross-cultural examination of hedonic and eudaimonic well-being. Psychological Assessment, 28(5), 471-482. https://doi.org/10.1037/pas0000209

Feather, N. T. (1996). Values, deservingness, and attitudes toward high achievers: Research on tall poppies. In C. Seligman, J. M. Olson, \& M. P. Zanna (Eds.), The Ontario symposium: The psychology of values. (Vol. 8, pp. 215-251). Lawrence Erlbaum Associates.

Fredrickson, B. L., Cohn, M. A., Coffey, K. A., Pek, J., \& Finkel, S. M. (2008). Open hearts build lives: positive emotions, induced through loving-kindness meditation, build consequential personal resources. Journal of Personality and Social Psychology, 95(5), 1045-1062. https://doi.org/10.1037/a0013262

Giménez, A. C., \& Tamajón, L. G. (2019). Analysis of the third-order structuring of Shalom Schwartz's theory of basic human values. Heliyon, 5(6), e01797. https://doi.org/10.1016/j.heliyon.2019.e01797

Giuntoli, L., Condini, F., Ceccarini, F., Huta, V., \& Vidotto, G. (2020). The different roles of hedonic and eudaimonic motives for activities in predicting functioning and well-being experiences. Journal of Happiness Studies, 1-15.

Grossmann, I., Oakes, H., \& Santos, H. C. (2019). Wise reasoning benefits from emodiversity, irrespective of emotional intensity. Journal of Experimental Psychology: General, 148(5), 805-823. https://doi. org/10.1037/xge0000543

Hayes, A. F. (2013). Methodology in the social sciences. Introduction to mediation, moderation, and conditional process analysis: A regression-based approach. New York, NY: Guilford Press.

Hayes, S. C., Strosahl, K., \& Wilson, K. G. (1999). Acceptance and commitment therapy: An experiential approach to behavior change. Guilford Press.

Henricksen, A., \& Stephens, C. (2010). An exploration of the happiness-enhancing activities engaged in by older adults. Ageing International, 35, 311-326. https://doi.org/10.1007/s12126-010-9059-y

Henricksen, A., \& Stephens, C. (2013). The happiness-enhancing activities and positive practices inventory (HAPPI): Development and validation. Journal of Happiness Studies, 14, 81-98. https://doi.org/10. 1007/s10902-011-9317-z

Hui, B. P., Ng, J. C., Berzaghi, E., Cunningham-Amos, L. A., \& Kogan, A. (2020). Rewards of kindness? A meta-analysis of the link between prosociality and well-being. Psychological Bulletin, 146(12), 10841116. https://doi.org/10.1037/bu10000298 
Huta, V., \& Ryan, R. M. (2010). Pursuing pleasure or virtue: The differential and overlapping well-being benefits of hedonic and eudaimonic motives. Journal of Happiness Studies, 11(6), 735-762. https:// doi.org/10.1007/s10902-009-9171-4

Huta, V., \& Waterman, A. S. (2014). Eudaimonia and its distinction from hedonia: Developing a classification and terminology for understanding conceptual and operational definitions. Journal of Happiness Studies: An Interdisciplinary Forum on Subjective Well-Being, 15(6), 1425-1456. https://doi.org/10. 1007/s10902-013-9485-0

Hofstede Insights. (2018). Culture compass TM. Retrieved from: https://www.hofstede-insights.com/count ry-comparison/poland/.

Johns, G., \& Miraglia, M. (2015). The reliability, validity, and accuracy of self-reported absenteeism from work: A meta-analysis. Journal of Occupational Health Psychology, 20(1), 1-14. https://doi.org/10. 1037/a0037754

Joshanloo, M., \& Ghaedi, G. (2009). Value priorities as predictors of hedonic and eudaimonic aspects of well-being. Personality and Individual Differences, 47(4), 294-298. https://doi.org/10.1016/j.paid. 2009.03.016

Jost, J. T., \& Hunyady, O. (2002). The psychology of system justification and the palliative function of ideology. European Review of Social Psychology, 13, 111-153. https://doi.org/10.1080/1046328024 0000046

Kaczmarek, L. D. (2017a). Eudaimonic motivation. In V. Zeigler-Hill \& T. K. Shackelford (Eds.), Encyclopedia of personality and individual differences. New York, NJ: Springer.

Kaczmarek, L. D. (2017b). Hedonic motivation. In V. Zeigler-Hill \& T. K. Shackelford (Eds.), Encyclopedia of personality and individual differences. New York, NJ: Springer.

Karabati, S., \& Cemalcilar, Z. (2010). Values, materialism, and well-being: A study with Turkish university students. Journal of Economic Psychology, 31, 624-633. https://doi.org/10.1016/j.joep.2010.04.007

Kashdan, T., Biswas-Diener, R., \& King, L. (2008). Reconsidering happiness: The costs of distinguishing between hedonics and eudaimonia. Journal of Positive Psychology, 3(4), 219-233. https://doi.org/10. 1080/17439760802303044

Kim-Prieto, C., Diener, E., Tamir, M., Scollon, C., \& Diener, M. (2005). Integrating the diverse definitions of happiness: A time-sequential framework of subjective well-being. Journal of Happiness Studies, 6 , 261-300. https://doi.org/10.1007/s10902-005-7226-8

Kłym, M., Karaś, D., Najderska, M., \& Cieciuch, J. (2014, July). Polish version of the Questionnaire for Eudaimonic Well-Being (QEWB). Paper presented at the 28th International Congress of Applied Psychology, Paris, France.

Larsen, J. T., \& McGraw, A. P. (2011). Further evidence for mixed emotions. Journal of Personality and Social Psychology, 100(6), 1095. https://doi.org/10.1037/a0021846

Lee, J. A., Sneddon, J. N., Daly, T. M., Schwartz, S. H., Soutar, G. N., \& Louviere, J. J. (2019). Testing and extending Schwartz refined value theory using a best-worst scaling approach. Assessment, 26(2), 166-180. https://doi.org/10.1177/1073191116683799

Lewin, K. (1952). Constructs in field theory [1944]. In D. Cartwright (Ed.), Field theory in social science: Selected theoretical papers by Kurt Lewin. (pp. 30-42). Tavistock.

Maslow, A. H. (1954). Motivation and personality. Harper \& Brothers.

Oishi, S., Diener, E., Suh, E., \& Lucas, R. E. (1999). Value as a moderator in subjective well-being. Journal of Personality, 67(1), 157-184. https://doi.org/10.1111/1467-6494.00051

Park, N., Peterson, C., \& Seligman, M. E. (2004). Strengths of character and well-being. Journal of Social and Clinical Psychology, 23(5), 603-619. https://doi.org/10.1521/jscp.23.5.603.50748

Pearl, J. (2012). The causal mediation formula-A guide to the assessment of pathways and mechanisms. Prevention Science, 13, 426-436. https://doi.org/10.1007/s11121-011-0270-1

Pienaar, J. M., Beukes, R. B. I., \& Esterhuyse, K. G. F. (2006). The Relationship between conservatism and psychological well-being in adolescents. South African Journal of Psychology, 36(2), 391-406.

Quoidbach, J., Gruber, J., Mikolajczak, M., Kogan, A., Kotsou, I., \& Norton, M. I. (2014). Emodiversity and the emotional ecosystem. Journal of Experimental Psychology: General, 143(6), 2057-2066. https://doi.org/10.1037/a0038025

Roccas, S., \& Sagiv, L. (2010). Personal values and behavior: Taking the cultural context into account. Social and Personality Psychology Compass, 4, 30-41. https://doi.org/10.1111/j.1751-9004.2009. 00234.x

Rogers, C. R. (1951). Client-centered therapy: Its current practice, implications, and theory. Houghton Mifflin.

Rohan, M. J. (2000). A rose by any name? The values construct. Personality and Social Psychology Review, 4, 255-277. https://doi.org/10.1207/S15327957PSPR0403_4 
Ryan, R., \& Deci, E. (2000). Self-determination theory and the facilitation of intrinsic motivation, social development, and well-being. American Psychologist, 55(1), 68-78. https://doi.org/10.1037/0003066X.55.1.68

Sagiv, L., \& Schwartz, S. H. (2000). Value priorities and subjective well-being: Direct relations and congruity effects. European Journal of Social Psychology, 30, 177-198. https://doi.org/10.1002/(SICI)10990992(200003/04)30:2\%3c177::AID-EJSP982\%3e3.0.CO;2-Z

Sarkova, M., Bacikova-Sleskova, M., Orosova, O., Madarasova Geckova, A., Katreniakova, Z., Klein, D., \& van Dijk, J. P. (2013). Associations between assertiveness, psychological well-being, and self-esteem in adolescents. Journal of Applied Social Psychology, 43(1), 147-154. https://doi.org/10.1111/j.15591816.2012.00988.x

Schreiber, J. B., Nora, A., Stage, F. K., Barlow, E. A., \& King, J. (2006). Reporting structural equation modelling and confirmatory factor analysis results: A review. The Journal of Educational Research, 99(6), 323-338. https://doi.org/10.3200/JOER.99.6.323-338

Schutte, N. S., \& Malouff, J. M. (2019). The impact of signature character strengths interventions: A metaanalysis. Journal of Happiness Studies, 20(4), 1179-1196. https://doi.org/10.1007/s10902-018-9990-2

Schwartz, S. H. (1992). Universals in the content and structure of values: Theory and empirical tests in 20 countries. In M. Zanna (Ed.), Advances in experimental social psychology. (Vol. 25, pp. 1-65). Academic Press.

Schwartz, S. H. (2007). Value orientations: Measurement, antecedents and consequences across nations. Measuring attitudes cross-nationally: Lessons from the European Social Survey, 169, 204

Schwartz, S. H., \& Butenko, T. (2014). Values and behavior: Validating the refined value theory in Russia. European Journal of Social Psychology, 44(7), 799-813. https://doi.org/10.1002/ejsp.2053

Schwartz, S., Cieciuch, J., Vecchione, M., Davidov, E., Fischer, R., Beierlein, C., \& Konty, M. (2012). Refining the theory of basic individual values. Journal of Personality and Social Psychology, 103(4), 663-688. https://doi.org/10.1037/a0029393

Schwartz, S. H., \& Sortheix, F. M. (2018). Values and subjective well-being. In E. Diener, S. Oishi, \& L. Tay (Eds.), Handbook of well-being. Salt Lake City, UT: DEF Publishers. doi:nobascholar.com.

Seligman, M. E., Steen, T. A., Park, N., \& Peterson, C. (2005). Positive psychology progress: Empirical validation of interventions. American Psychologist, 60(5), 410-421. https://doi.org/10.1037/0003-066X. 60.5 .410

Sheldon, K. M., \& Krieger, L. S. (2014). Walking the talk: Value importance, value enactment, and wellbeing. Motivation and Emotion, 38(5), 609-619. https://doi.org/10.1007/s11031-014-9424-

Sortheix, F. M., \& Lönnqvist, J. (2015). Person-group value congruence and subjective well-being in students from Argentina, Bulgaria and Finland: The role of interpersonal relationships. Journal of Community \& Applied Social Psychology, 25(1), 34-48. https://doi.org/10.1002/casp.2193

Sortheix, F. M., \& Schwartz, S. H. (2017). Values that underlie and undermine well-Being: Variability across countries. European Journal Of Personality, 31(2), 187-201. https://doi.org/10.1002/per.2096

Van Hiel, A., \& Brebels, L. (2011). Conservatism is good for you: Cultural conservatism protects selfesteem in older adults. Personality and Individual Differences, 50(1), 120-123. https://doi.org/10. 1016/j.paid.2010.09.002

Van Hiel, A., \& De Clercq, B. (2009). Authoritarianism is good for you: Right-wing authoritarianism as a buffering factor for mental distress. European Journal of Personality, 23(1), 33-50. https://doi.org/10. 1002/per.702

Vecchione, M., Schwartz, S. H., Caprara, G. V., Schoen, H., Cieciuch, J., Silvester, J., \& Alessandri, G. (2015). Personal values and political activism: A cross-national study. British Journal of Psychology, 106(1), 84-106. https://doi.org/10.1111/bjop.12067

Waterman, A. S. (1990). The relevance of Aristotle's conception of eudaimonia for the psychological study of happiness. Theoretical and Philosophical Psychology, 10, 39-44. https://doi.org/10.1037/h0091489

Waterman, A. S. (1990). Personal expressiveness: Philosophical and psychological foundations. Journal of Mind and Behavior, 11, 47-74

Waterman, A. S., Schwartz, S. J., Zamboanga, B. L., Ravert, R. D., Williams, M. K., Bede Agocha, V., \& Brent Donnellan, M. (2010). The Questionnaire for Eudaimonic well-being: Psychometric properties, demographic comparisons, and evidence of validity. Journal of Positive Psychology, 5(1), 41-61. https://doi.org/10.1080/17439760903435208

Watson, D., Clark, L. A., \& Tellegen, A. (1988). Development and validation of brief measures of positive and negative affect: The PANAS scales. Journal of Personality and Social Psychology, 54, 1063-1070. https://doi.org/10.1177/1073191108328890

Publisher's Note Springer Nature remains neutral with regard to jurisdictional claims in published maps and institutional affiliations. 